Revista Destaques Acadêmicos, Lajeado, v. 8, n. 3, 2016. ISSN 2176-3070 DOI: http://dx.doi.org/10.22410/issn.2176-3070.v8i3a2016.1053 www.univates.br/revistas

\title{
INCIDÊNCIA DE ENTEROPARASITOS INTESTINAIS EM UMA ESCOLA INFANTIL PÚBLICA E UMA ESCOLA INFANTIL COMUNITÁRIA, EM UM MUNICÍPIO NO INTERIOR DO RIO GRANDE DO SUL
}

\author{
Alisson André Gross ${ }^{1}$, Gabriela Kniphoff da Silva ${ }^{2}$
}

\begin{abstract}
Resumo: As infecções parasitárias intestinais, ao longo dos anos, se tornaram um grande problema de saúde pública, com altas taxas de prevalência. As crianças são mais afetadas, devido à sua imaturidade imunológica e maus hábitos de higiene. $\mathrm{O}$ objetivo do trabalho consiste em analisar a incidência de parasitos intestinais em uma escola infantil pública e uma escola infantil comunitária, em um município no interior do Rio Grande do Sul. Foram analisadas 123 amostras, seguindo o método de Hoffman, em agosto e setembro de 2015, sendo 25 crianças da rede pública e 16 da comunitária. Cada uma realizou três coletas. Entre as crianças da rede pública, duas (8\%) estavam parasitadas, uma com Ascaris lumbricoides e outra com Entamoeba coli. Já na rede comunitária, uma (6,25\%) amostra se apresentou positiva para Giardia lamblia. Concluise que existe uma baixa incidência de parasitos intestinais, provavelmente devido às boas condições socioeconômicas, ambientais e de saneamento básico da cidade.
\end{abstract}

Palavras-chave: Parasitoses. EPF. Crianças. Escola infantil.

\section{INTRODUÇÃO}

Desde a publicação de "This Wormy World", a primeira revisão sistemática sobre o impacto do parasitismo em humanos, em 1947, por Norman Stoll, os esforços globais aumentaram consideravelmente para enfrentar o parasitismo nos humanos (WHO, 2012). As parasitoses são doenças causadas por parasitos que vivem no interior ou no exterior de outros hospedeiros. Essa associação nem sempre é prejudicial, mas permite que o parasito extraia seu alimento e obtenha abrigo (CIMERMAN; CIMERMAN, 1999). Os helmintos

1 Biomédico. E-mail: agross@univates.br.

2 Biomédica, mestre e doutoranda em Genética e Biologia Molecular pela UFRGS, professora da UNIVATES, Lajeado, RS, Brasil. E-mail: gkdsilva@univates.br. 
estão entre os agentes parasitários mais comuns que afetam as populações mais marginalizadas, de baixa renda e com poucos recursos (WHO, 2012).

No mundo, estima-se que cerca de $25 \%$ das doenças infeciosas sejam causadas por protozoários e/ou helmintos, sendo mais comuns em países mais pobres, e contribuindo com cerca de 200.000 mortes por ano (KUBIAK et al., 2015). Mesmo que nos países industrializados tenham sido praticamente erradicadas, cerca de 2 bilhões de pessoas, principalmente crianças, estão parasitadas. Essas doenças persistem devido a fatores socioeconômicos, ambientais, falta de higiene, acesso à água potável, clima, políticas públicas como prevenção e tratamento e hábitos nutricionais (ADEKOLUJO et al., 2015; ZAISS et al., 2015).

Diversas medidas são sugeridas para uma redução significativa destas doenças. Entre elas, podem-se realizar tratamentos em massa da população, reduzindo o número de portadores e, consequentemente, reduzindo o ciclo do parasito, ou até mesmo, eliminando-o, quando o hospedeiro principal é o homem (NETO et al., 2008). O controle das parasitoses também pode ser feito através de melhorias nas condições de saneamento, métodos seguros de descarte das fezes, água de abastecimento e uma boa atenção à educação em saúde. O objetivo em longo prazo é diminuir a prevalência, a intensidade e a gravidade, reduzindo os níveis a números significantes. O processo é relativamente lento, gera altos investimentos e acaba não sendo utilizado com o tempo (NORHAYATI et al., 2003).

As enteroparasitoses constituem um grave problema de saúde pública em países em desenvolvimento, assim como no Brasil. O ambiente quente dos países tropicais, associado a múltiplos fatores, como a desnutrição, contaminação da água e alimentos, condições sanitárias, grau de escolaridade, presença de reservatórios e vetores, promovem o desenvolvimento e a propagação dos helmintos e protozoários (MACHADO et al., 1999; KOMAGOME et al., 2007; BASSO et al., 2008). As parasitoses trazem diversos impactos na saúde da população acometida, incluindo a má nutrição, principalmente em crianças com idade escolar, o que acaba dificultando o aprendizado e o desenvolvimento físico. Em comparação aos adultos, as crianças tendem a ter mais chances de infecção, justamente por serem mais ativas fisicamente, por raramente empregarem bons hábitos de higiene e por terem um sistema imunológico ainda em desenvolvimento (KUBIAK et al., 2015).

Estudos demonstram que as crianças que frequentam as creches adoecem mais, com prevalência de doenças infecciosas, se comparadas às que são cuidadas exclusivamente em casa. Estima-se que aproximadamente $55,3 \%$ das crianças no Brasil apresentam infecção por enteroparasitos, sendo frequentemente associados à diarreia crônica e desnutrição, demonstrando que as crianças que frequentam ambientes de maior coletividade, como as creches, são mais acometidas pela infecção (PEDRAZA; QUEIROZ; SALES, 2014). 
Dessa forma, as escolas infantis são um importante local de investigação dos parasitos, pois nem sempre os professores conseguem ter um controle sobre seus alunos, pelo fato de serem muitos para os seus cuidados. Com isso, a higiene das crianças pode não ser feita de forma adequada. Diante dessa situação, considera-se de grande importância a investigação da incidência de parasitoses em qualquer município, estado ou país para, assim, conhecer a atual situação desse local, e pensar em programas de tratamento e prevenção, a fim de diminuir a incidência de casos, e melhorar a qualidade de vida da população. Logo, o presente estudo teve por objetivo verificar a incidência de parasitoses intestinais em uma escola infantil pública e uma escola infantil comunitária, em um município no interior do Rio Grande do Sul.

\section{METODOLOGIA}

As amostras foram coletadas no mês de agosto e setembro de 2015, de crianças que frequentaram uma escola de Educação Infantil Pública e uma Comunitária, de ambos os sexos, oriundas de um município do interior do Rio Grande do Sul, totalizando um número de 41 participantes, sendo que 25 eram da rede pública e 16 da rede comunitária. Foram realizadas três coletas de cada criança, com intervalos de um dia, totalizando 123 amostras. Juntamente com as coletas, os pais responderam um questionário, com questões pertinentes ao estudo.

As coletas foram realizadas pelos pais, com instruções prévias, em potes estéreis, fornecidos pelo pesquisador, sendo armazenadas em geladeira e transportadas até o laboratório de Ensino de Parasitologia da Univates.

Foram incluídas na pesquisa crianças de 0 a 5 anos de idade, que residiam no município estudado, cujos pais assinaram o Termo de Consentimento Livre e Esclarecido (TCLE).

Os exames foram realizados seguindo o método de Hoffman, Pons e Janer ou Lutz, para visualizar cistos de protozoários, ovos e larvas de helmintos. A técnica tem como fundamento um sistema de sedimentação espontânea dos parasitos, com posterior identificação microscópica das formas parasitárias (CARLI, 1994). Todas as amostras foram processadas e analisadas nos laboratórios de Ensino de Parasitologia e Microscopia da Univates.

O projeto foi submetido e aprovado pelo Comitê de Ética em Pesquisa da Univates em agosto de 2015 (parecer 1.154.518).

\section{RESULTADOS}

Das 25 crianças que fazem parte da rede municipal, 56\% são do sexo masculino e $44 \%$ feminino, tendo idades que variam de recém-nascidos a 5 anos, sendo que $96 \%$ moram na zona urbana da cidade. Dentre esses, $40 \%$ nunca fizeram exame parasitológico de fezes (EPF). Dentre os participantes, $24 \%$ informaram que tomaram algum medicamento antiparasitário no último 
ano e $4 \%$ já visualizaram algo parecido com vermes nas fezes. Das 25 crianças, apenas duas estavam parasitadas, uma com Ascaris lumbricoides e outra com Entamoeba coli, que é considerada comensal e não patogênica, correspondendo a $8 \%$ de amostras positivas.

$\mathrm{Na}$ escola da rede comunitária, participaram 16 crianças entre 3 e 5 anos de idade, sendo $50 \%$ do sexo feminino e $50 \%$ do masculino, com $93,75 \%$ morando na zona urbana. Entre todos os participantes, 56,25\% já realizaram exame parasitológico de fezes, sendo que 56,25\% fizeram tratamento antiparasitário no último ano e 6,25\% já visualizou algo parecido com vermes nas fezes dos seus filhos. Entre as 16 crianças que participaram do estudo, apenas uma estava parasitada, sendo o parasita identificado como Giardia lamblia, correspondendo a $6,25 \%$ de amostras positivas.

Tabela 1 - Dados obtidos a partir do questionário aplicado aos pais

\begin{tabular}{|c|c|c|}
\hline & Escola Pública & Escola Comunitária \\
\hline \multicolumn{3}{|c|}{ 1. Abastecimento de água } \\
\hline Corsan & $3(12 \%)$ & $1(6,25 \%)$ \\
\hline Prefeitura & $22(88 \%)$ & $10(62,5 \%)$ \\
\hline Associação da água & $0(0 \%)$ & $3(18,75 \%)$ \\
\hline Poço artesiano & $0(0 \%)$ & $2(12,5 \%)$ \\
\hline \multicolumn{3}{|c|}{ 2. Lavagem dos alimentos } \\
\hline Água & $23(92 \%)$ & $15(93,75 \%)$ \\
\hline Água e vinagre & $2(8 \%)$ & $1(6,25 \%)$ \\
\hline \multicolumn{3}{|c|}{ 3. Origem dos alimentos } \\
\hline Mercado & $21(67,7 \%)$ & $16(76,2 \%)$ \\
\hline Feira & $4(12,9 \%)$ & $2(9,5 \%)$ \\
\hline Horta & $4(12,9 \%)$ & $3(14,3 \%)$ \\
\hline Vendedor & $2(6,5 \%)$ & $0(0 \%)$ \\
\hline \multicolumn{3}{|c|}{ 4. Contato com animais } \\
\hline Sim & $17(68 \%)$ & $9(56,25 \%)$ \\
\hline Não & $8(32 \%)$ & $7(43,75 \%)$ \\
\hline \multicolumn{3}{|l|}{ 5. Quais? } \\
\hline Cachorro & $15(68,2 \%)$ & $8(66,67 \%)$ \\
\hline Gato & $4(18,2 \%)$ & $3(25,0 \%)$ \\
\hline Galinha & $2(9,1 \%)$ & $1(8,33 \%)$ \\
\hline Coelho & $1(4,5 \%)$ & $0(0 \%)$ \\
\hline
\end{tabular}

Fonte: Do autor. 


\section{DISCUSSÃO}

Estima-se que cerca de 1,7 bilhões de novos casos de diarreia surgem a cada ano, sendo muitas vezes devastadores para as crianças menores de 5 anos, continuando a ser uma das principais causas de morbidade e mortalidade, globalmente. Segundo a Organização Mundial da Saúde, a diarreia é caracterizada por fezes moles ou líquidas, por pelo menos três vezes ao dia, bastante comum em infecções gastrointestinais, causadas principalmente por parasitos intestinais. Essas infecções são frequentes nos países em desenvolvimento, tendo os protozoários mais comuns a Giardia lamblia, Cryptosporidium parvum e Entamoeba histolytica, com sintomas que variam desde a diarreia com cólicas abdominais, flatulência e perda de peso, até o óbito do paciente (MULATU et al., 2015).

Os resultados desta pesquisa encontraram uma positividade abaixo da média de parasitos, nas crianças de ambas as escolas analisadas, sendo o Ascaris lumbricoides um dos parasitos identificados. A ascaridíase pode causar sérias complicações, tais como obstrução intestinal e biliar, abscesso do fígado, pancreatite, intolerância a lactose, má absorção de vitamina A e outros nutrientes. Mas a complicação mais comum é a obstrução intestinal em crianças, que incluem oclusão ou suboclusão intestinal quando chegam ao íleo, através da compactação dos vermes, uma vez que o lúmen é pequeno, provocando o chamado "bolo de áscaris", que pode levar ao infarto ou perfuração intestinal (BETHONY et al., 2006; NETO et al., 2008; HEFNY; SAADELDIN; ABUZIDAN, 2009).

Também foram visualizados, neste estudo, cistos de Entamoeba coli em uma criança e de Giardia lamblia, em outra criança. A contaminação por Giardia lamblia e Entamoeba coli se dá através de cistos presentes na água e nos alimentos, e também de pessoa a pessoa, principalmente em lugares com pouca higiene e precárias condições sanitárias, facilitando o contato com as formas infectantes, principalmente nas creches (PEDRAZA; QUEIROZ; SALES; 2014). O mesmo ocorre no caso da ascaridíase, sendo que sua transmissão pode ocorrer através da ingestão de ovos infectantes provenientes do solo, alimentos ou água contaminada com fezes humanas (NEVES et al., 2000; FUNG; CAIRNCROSS, 2009).

Os resultados desta pesquisa divergem de outros estudos realizados com amostras de crianças, que encontraram uma positividade muito maior. Um estudo realizado em Belo Horizonte, no ano de 2006, mostrou que 24,6\% das 472 crianças analisadas estavam parasitadas. Sendo que o parasito de maior prevalência foi a Entamoeba coli, demonstrando fortes indícios de precariedade nas condições sanitárias da população. A Giardia lamblia também foi evidenciada com alta prevalência, comprovando sua existência em creches (MENEZES et al., 2008). 
No estado de São Paulo, na cidade de Botucatu, o coeficiente geral de enteroparasitos foi de 53,4\%, sendo o parasito de maior prevalência a Giardia lamblia, presente em $26,88 \%$ das 279 amostras de crianças (CARVALHO; CARVALHO; MASCARINI, 2006). Ambos os estudos encontraram uma alta frequência de parasitos, em relação aos resultados deste estudo, e isso pode ser explicado principalmente pelas más condições de saneamento básico do local, assim como também pelo tamanho da população e da cidade, que é muito maior, dificultando um trabalho de prevenção de melhor qualidade. Frequentemente, em áreas mais pobres da cidade há carência de informações sobre higiene pessoal e lavagem dos alimentos, lembrando que muitas vezes também não há acesso a uma água de boa qualidade, resultando em mais casos de parasitoses.

A partir do questionário aplicado no presente estudo, foi possível relacionar a qualidade da água de abastecimento nas residências ao nível de contaminação das amostras. Os resultados indicam que o tratamento realizado pelas distribuidoras de água está sendo feito de forma adequada, já que os resultados obtidos na pesquisa foram baixos, mas outro fator que também contribui é a lavagem dos alimentos que na maioria dos casos era feita somente com água, mas se feita de forma adequada e com uma água de qualidade, a contaminação se torna relativamente baixa, indicando boas condições de saneamento.

A boa estrutura das duas escolas, assim como a organização, a limpeza e o comprometimento dos professores com a higiene das crianças são fatores importantes que contribuem com a baixa incidência de parasitos encontrada.

Vale ressaltar que o estudo não reflete a realidade do Brasil, apenas de uma pequena cidade do interior do Rio Grande do Sul, já que o Brasil é um país com muitas diferenças sociais, econômicas e culturais. Os dados levantados estão de acordo com outro estudo realizado em uma cidade de pequeno porte, no município de Estiva Gerbi, São Paulo, que nos anos de 2000 a 2002, também encontrou uma baixa positividade, de apenas 1,5\% para Ascaris lumbricoides, $0,1 \%$ para Trichuris trichiura e $0,1 \%$ para ancilostomídeos em crianças entre 0 a 7 anos, residentes em área urbana e rural (FONSECA et al., 2010).

\section{CONCLUSÃO}

Conclui-se que existe uma incidência baixa de parasitos intestinais se comparado a outros estudos semelhantes, tanto na escola da rede municipal, quanto na rede comunitária, e esses dados são indicativos de boas condições socioeconômicas, ambientais e de saneamento básico, que são fundamentais para evitar uma das principais formas de transmissão dos parasitos, no caso a água e os alimentos. O bom estado das escolas e a prática aplicada aos cuidados em higiene também reforçam a baixa incidência de parasitos nas crianças. 


\section{REFERÊNCIAS}

ADEKOLUJO, Daniel R. et al. Poliovirus and other enteroviruses in children infected with intestinal parasites in Nigeria. J. Infect. Dev. Ctries., Italy, v. 9, n. 10, p. 11661171, April. 2015. Disponível em: < http:/ / www.jidc.org/index.php/journal/article/ view/26517494/1406>. Acesso em: 22 nov. 2015.

BETHONY, J. et al. Soil-transmitted helminth infections: ascariasis, trichuriasis, and hookworm. The Lancet, London, v. 367, n. 9521, p. 1521-1532, May. 2006.

Disponível em: <http:/ / www.sciencedirect.com.sci-hub.org/science/article/pii/ S0140673606686534>. Acesso em: 04 jun. 2015.

CARLI, G. A. de. Diagnóstico laboratorial das parasitoses humanas: métodos e técnicas. Rio de Janeiro: MEDSI, 1994.

CARVALHO, T. B. de; CARVALHO, L. R. de; MASCARINI, L. M. Occurrence of enteroparasites in day care centers in Botucatu (São Paulo State, Brazil) with emphasis on Cryptosporidium sp., Giardia duodenalis and Enterobius vermicularis. Rev. Inst. Med. trop. S. Paulo, São Paulo, v. 48, n. 5, p. 269-273, Oct. 2006.

Disponível em: <http:/ / www.scielo.br/scielo.php?script=sci_arttext\&pid=S0036$46652006000500006 \& l n g=e n \& n r m=i s o>$. Acesso em: 26 abr. 2015.

CIMERMAN, B.; CIMERMAN, S. Parasitologia humana e seus fundamentos gerais. São Paulo: Atheneu, 1999.

FONSECA, E. O. L. et al. Prevalência e fatores associados às geo-helmintíases em crianças residentes em municípios com baixo IDH no Norte e Nordeste brasileiros. Cad. Saúde Pública, Rio de Janeiro, v. 26, n. 1, p. 143-152, Jan. 2010. Disponível em: <http:/ / www.scielo.br/scielo.php?script=sci_arttext\&pid=S0102-311X2010000100015 \&lng=en\&nrm=iso $>$. Acesso em: 25 nov. 2015.

FUNG, I. C-H.; CAIRNCROSS, S. Ascariasis and handwashing. Transactions of the Royal Society of Tropical Medicine and Hygiene, Oxford, v. 103, n. 3, p. 215-222, March. 2009. Disponível em: <http:/ / www.sciencedirect.com.sci-hub.org/science/ article/pii/S0035920308003416>. Acesso em: 24 maio 2015.

HEFNY, A.. F; SAADELDIN, Y., A; ABU-ZIDAN, A. F. Management algorithm for intestinal obstruction due to ascariasis: a case report and review of the literature. Turkish Journal of Trauma \& Emergency Surgery, Al Ain, v. 15, n. 3, p. 301305, May. 2009. Disponível em: <http:/ / www.journalagent.com/travma/pdfs / UTD_15_3_301_305.pdf $>$. Acesso em: 17 maio 2015.

KOMAGOME, S. H. et al. Fatores de risco para infecção parasitária intestinal em crianças e funcionário de creches. Cienc. Cuid. Saúde, Maringá, v. 6, supl. 2, p. 442447, Mar. 2007. Disponível em: <http:/ / www.periodicos.uem.br/ojs/index.php/ CiencCuidSaude/article/view/5354/3391>. Acesso em: 03 maio 2015.

KUBIAK, K. et al. The Prevalence of intestinal parasites in children in preschools and orphanages in the Warmia-Masuria Province (North-Eastern Poland). Przegl 
Epidemiol, Warszava, v. 69, n. 3, p. 483-488, 2015. Disponível em: <http:/ /www.ncbi. nlm.nih.gov/pubmed/26519844>. Acesso em: 15 nov. 2015.

MACHADO, Renato C. et al. Giardíase e helmintíases em crianças de creches e escolas de $1^{\circ}$ e $2^{\circ}$ graus (públicas e privadas) da cidade de Mirassol (SP, Brasil). Rev. Soc.

Bras. Med. Trop., Uberaba, v. 32, n. 6, p. 697-704, Dec. 1999. Disponível em: <http:/ / www.scielo.br/scielo.php?script=sci_arttext\&pid=S0037-86821999000600013\&lng=en \&nrm=iso >. Acesso em: 03 maio 2015.

MENEZES, A. L. et al. Prevalence of intestinal parasites in children from public daycare centers in the city of Belo Horizonte, Minas Gerais, Brazil. Rev. Inst. Med. trop. S. Paulo, São Paulo, v. 50, n. 1, p. 57-59, Feb. 2008. Disponível em: <http:/ /www. scielo.br/scielo.php?script=sci_arttext\&pid=S0036-46652008000100013\&lng=en\&nrm= iso>. Acesso em: 26 abr. 2015.

MULATU G. Z. et al. Intestinal parasitic infections among children under five years of age presenting with diarrhoeal diseases to two public health facilities in Hawassa, South Ethiopia. Infectious Diseases of Poverty, London, v. 49, n 4, p. 1-8, Nov. 2015. Disponível em: http:/ /www.ncbi.nlm.nih.gov/pmc/articles/PMC4632267/.>. Acesso em: 26 nov. 2015.

NETO, V. A. et al. Parasitologia: uma abordagem clínica. Rio de Janeiro: Elsevier, 2008 .

NEVES, D. P. Parasitologia humana. 10. ed. São Paulo: Atheneu, 2000.

NORHAYATI, M. et al. Intestinal Parasitic Infections In Man: A Review. Med J Malaysia, Kuala Lumpur, v. 58, n. 2, p. 296-235, June. 2003. Disponível em: <http:/ / www.e-mjm.org/2003/v58n2/Intestinal_Parasitic_Infections.pdf >. Acesso em: 17 maio 2015.

PEDRAZA, D. F; QUEIROZ, D. de; SALES, M. C. Doencas infecciosas em crianças pre-escolares brasileiras assistidas em creches. Ciênc. saúde coletiva, Rio de Janeiro, v. 19, n. 2, p. 511-528, Feb. 2014. Disponível em: <http:/ / www.scielosp.org/scielo. php?script=sci_arttext\&pid=S1413-81232014000200511\&lng=en\&nrm=iso $>$. Acesso em: 25 nov. 2015.

WORLD HEALTH ORGANIZATION. World Health Report 2012. Geneva: WHO; 2012.

ZAISS, M. M. et al. The Intestinal Microbiota Contributes to the Ability of Helminths to Modulate Allergic Inflammation. Immunity, Cambridge v. 43, p. 1-13, Oct. 2015. Disponível em: <http:/ / www.cell.com/immunity/abstract/ S1074-7613\%2815\%2900397-0?_returnURL=http\%3A\%2F\%2Flinkinghub.elsevier. com\%2Fretrieve \%2Fpii\%2FS1074761315003970\%3Fshowall\%3Dtrue>. Acesso em: 15 nov. 2015. 